\title{
LncRNA KRT19P3 Is Involved in Breast Cancer Cell Proliferation, Migration and Invasion
}

\author{
Yanping Fan ${ }^{1,2 \dagger}$, Xiaotong Dong ${ }^{1,2 \dagger}$, Meizeng $\mathrm{Li}^{1,2}$, Pengju $\mathrm{Liu}^{3}$, Jie Zheng ${ }^{2}$, Hongli $\mathrm{Li}^{2}$ \\ and Yunxiang Zhang ${ }^{1 *}$ \\ 1 Pathology Department, First Affiliated Hospital of Weifang Medical University (Weifang People's Hospital), Weifang, China, \\ 2 Department of Basic Medicine, Weifang Medical University, Weifang, China, ${ }^{3}$ School of Economics, Qingdao University, \\ Qingdao, China
}

OPEN ACCESS

Edited by:

Min Tang,

Jiangsu University, China

Reviewed by:

Lina Zhao,

Chinese Academy of Medical Sciences, China Kebo LV,

Ocean University of China, China

*Correspondence: Yunxiang Zhang zhangbing199592@163.com

${ }^{\dagger}$ These authors have contributed equally to this work

Specialty section: This article was submitted to Cancer Imaging and Image-directed Interventions, a section of the journal

Frontiers in Oncology

Received: 21 October 2021 Accepted: 08 December 2021

Published: 04 January 2022

Citation:

Fan Y, Dong X, Li M, LiU P, Zheng J, Li $H$ and Zhang $Y$ (2022) LnCRNA KRT19P3 Is Involved in Breast Cancer Cell Proliferation, Migration and Invasion.

Front. Oncol. 11:799082. doi: 10.3389/fonc.2021.799082
Long non-coding RNAs (LncRNAs) have already been taken as critical regulatory molecules in breast carcinoma (BC). Besides, the progression of $\mathrm{BC}$ is closely associated with the immune system. However, the relationship between IncRNAs and the tumor immune system in BC has not been fully studied. LncRNA KRT19P3 has been reported to inhibit the progression of gastric cancer. In the present study, we first discovered that KRT19P3 was downregulated in BC tissues compared with para cancer tissue. Then we showed that KRT19P3 could be used as a marker to differentiate BC from para cancer tissue. Increased expression of KRT19P3 markedly inhibited the proliferation, migration, and invasion rate of $\mathrm{BC}$ cells in vitro and tumor growth of BC in vivo. Conversely, KRT19P3 knockdown by siRNA markedly promoted the proliferation, migration, and invasion rate of $\mathrm{BC}$ cells after being transfected. Comparison of clinical parameters showed an inverse relationship between the expression of KRT19P3 and pathological grade. Furthermore, immunohistochemistry $(\mathrm{IHC})$ was applied to reveal the positive rate of the expression of Ki-67, programmed death-ligand 1 (PD-L1), and CD8 in $\mathrm{BC}$ tissues. Correlation analysis showed that $\mathrm{Ki}-67$ and PD-L1 were inversely proportional to KRT19P3 but CD8 was directly proportional to KRT19P3. In conclusion, this study demonstrated that IncRNA KRT19P3 inhibits BC progression, and may affect the expression of PD-L1 in BC, which in turn affects CD8 ${ }^{+} \mathrm{T}$ (CD8 positive Cytotoxic $\mathrm{T}$ lymphocyte) cells in the immune microenvironment.

Keywords: breast cancer, long non-coding RNA, KRT19P3, PD-L1, CD8+ T cell, immune

\section{INTRODUCTION}

$\mathrm{BC}$ ranks the first in the diagnosis of female tumors and it is the fifth leading cause of death due to cancer worldwide (1-3). Despite advances in multiple types of treatments, the prognosis for BC patients remains dismal (4). For this reason, the identification of the mechanisms responsible for the pathogenesis of $\mathrm{BC}$ is urgent for improving the clinical outcome $(5,6)$.

LncRNAs are a kind of non-coding RNAs with methylguanosine cap and polyadenylate (poly-A) structure and a length of more than 200 nucleotides, most of which are transcripts of RNA 
polymerase II (7-9). At first, LncRNAs have been considered as non-functional genes. Nevertheless, with more deeply research, it has been discovered that lncRNAs play crucial roles in the progression of tumors by promoting or inhibiting biological behaviors such as cell proliferation, migration, and EMT (1014), which allows tumor and other types of cells around the tumor microenvironment to interact with each other. Meanwhile, LncRNAs can regulate the expression of key genes associated with immune function affecting the function of immune cells involved in the microenvironment of tumors (15). For instance, IncRNA KCNQ1OT1 promoted the progression of prostate cancer by suppressing $\mathrm{CD}^{+} \mathrm{T}$ cells cytotoxicity through the KCNQ1OT1/miR-15a/PD-L1 axis (16). Yilong Ai et al. found that CRNDE specifically sponged miR-545-5p to induce T-cell immunoglobulin and mucin domain-3 (TIM-3), thus contributing to $\mathrm{CD}^{+} \mathrm{T}$-cell exhaustion in Oral squamous cell carcinoma (OSCC) (17). Although the functional roles of lncRNAs in BC are diverse, the study of the relationship between lncRNAs and the tumor immune system is still in its infancy. T cell-regulated adaptive immune responses can induce the expression of PD-L1 in the tumor microenvironment (18). However, the expression of PDL1 inhibits T lymphocytes from playing a role in the immune microenvironment (7). From this point of view, it is interesting that lncRNAs are involved in the immune process.

LncRNA KRT19P3 is a pseudogene located on Chromosome 4. It was reported to inhibit gastric cancer proliferation and invasion through the NF- $\mathrm{BB}$ signaling pathway (19). To study the role of KRT19P3 in BC, we have investigated the function of KRT19P3 in BC in vitro and its association with the immune. This study provides a theoretical and experimental basis for immune-related studies between lncRNA KRT19P3 and BC.

\section{MATERIALS AND METHODS}

\section{Cell Culture}

BC cell line MDA-MB-231 was purchased from Procell Life Science \& Technology Co., Ltd. (Wuhan, China). The cell line was cultured in RPM1640 medium (Solarbio, USA) added with $10 \%$ FBS (Hyclone, Logan, UT, USA). The environment in the incubator was maintained at 5\% CO2 and a constant temperature of $37^{\circ} \mathrm{C}$.

\section{Reverse Transcription-Quantitative Real-Time PCR}

Axygen Total RNA Preparation Kit (Corning Life Sciences Co., Ltd., Suzhou, China) was used to extract total RNA. M-MuLV reverse transcriptase (New England Biolabs), RNase inhibitors, and dNTPs (Takara) was used to synthesize cDNA. A fluorescence quantitative PCR instrument (ABI7500Fast, USA) was used for RT-qPCR. GAPDH was used as an internal control.

The primer sequences are as follows:

GAPDH forward primer 5'-GCACCGTCAA-GGCT GAGAAC-3';

Reversed primer 5'-TGGTGAAGACGCCAGTGGA-3';
KRT19P3 forward primer5'-CAGTGAGAGGCAG AATCAGG-3';

Reversed primer5'-TTGGAGGTGGACAGGCTATT-3'.

The relative expression of KRT19P3 was analyzed by $2^{-\triangle \mathrm{CT}}$.

\section{Cell Transfection}

The pcDNA3.1-KRT19P3 plasmids and siRNAs were provided by Professor Jie Zheng, Department of Pathology, Weifang Medical College. Lipofectamine2000 (Invitrogen, USA) was used to transfect MDA-MB-231 cells. The plasmids and Lipofectamine 2000 Reagent were mixed and incubated in a 1: 1 ratio for 15 minutes and then added to a six-well plate. The siRNAs and Lipofectamine 2000 Reagent were mixed and incubated in a 1: 1.3 ratio for 15 minutes and then added to a six-well plate. The overexpression and knockdown efficiency was confirmed $24 \mathrm{~h}$ after transfection by qRT-PCR.

\section{EdU Assay}

MDA-MB-231 proliferation capacity was detected by BeyoClick $^{\mathrm{TM}}$ EdU-594 Cell Proliferation Assay Kit (Shanghai Biyuntian Biotechnology Co., Ltd., Shanghai, China). Cells after transfection $24 \mathrm{~h}$ were seeded onto 96 -well plates, and three duplicate wells were set for each group, $2 \times 10^{4}$ cells per well. Proliferation efficiency was the percentage of positive cells stained with EdU.

\section{Migration and Invasion Assay}

The potential of invasion and migration was detected using transwell inserts (Corning, Cambridge, MA, USA). In the migration assay, $4 \times 10^{4}$ cells were added above the chamber, RPMI1640 with 10\% FBS in the lower chamber, and the chambers were fixed after 24 hours. Apodemsa staining solution (Solarbio, USA) was stained for 40 minutes and observed microscopically. Invasion assay was performed by adding Matrigel (Corning, Cambridge, MA, USA) on the top of the membrane to simulate cell transmembrane assay. We randomly took five fields for cell counting under the microscope.

\section{In Vivo Tumorigenic}

Lentivirus vector LV-KRT19P3 and LV-NC transfected MDAMB-231 cells. $1 \times 10^{7}$ cells in $100 \mu$ l of PBS were injected into the mammary fat pads of 4-week-old female athymic BALB/c nude mice (Vital River, Beijing, China). Mice were grouped (six for each group) and housed under SPF conditions at the Experimental Animal Center of Weifang Medical College. Tumor growth was observed weekly and tumor volumes (V) were calculated as $\mathrm{V}=($ tumor length $\times$ width2)/2. Six weeks later, bioluminescence was used in the IVIS system. These mice were killed and tumor nodules were extracted and evaluated with hematoxylin and eosin staining. Animal use in the study was performed following the animal care and ethical committee of the medical sector in Weifang Medical College.

\section{Human tissues}

A total of 98 BC tissues and non-tumor breast tissues $5 \mathrm{~cm}$ away from the cancer region were obtained from the First Affiliated Hospital of Weifang Medical University from Feb 2019 to Jun 2019. 
Half of the tumor tissues were paraffin-embedded, and the other half tissues were stored at $-150^{\circ} \mathrm{C}$ for RT-qPCR detection. The above specimens have been diagnosed as BC by clinicians. This study conformed to the standards of the Ethics Committee of Weifang People's Hospital.

\section{Immunohistochemistry}

The expression of $\mathrm{CD}^{+} \mathrm{T}$ and $\mathrm{PD}-\mathrm{L} 1$ genes in 80 breast cancer tissues and matching normal tissue specimens were detected by IHC. Xylene was used to deparaffinized and diluted graded alcohols to hydrated; $\mathrm{pH} 9.0$ citric acid buffer was used to repair antigens at high temperature and pressure; endogenous peroxidase was blocked with $0.3 \% \mathrm{H}_{2} \mathrm{O}_{2}$; and incubated with primary antibodies of CD8 and PD-L1 (Beijing Zhongshan Jinqiao, China) for an hour at room temperature. Finally, DAB for color staining. The positive side of CD8 protein staining was mainly localized on the nucleus, and the experimental results were judged according to the proportion of positive cells, with $10 \%$ as the cutoff value, $\geq 10 \%$ as positive, and $<10 \%$ as negative.

\section{Statistical Analysis}

SPSS 25 software was used for statistical analysis. Graph Pad Prism7 software was used for picture drawing. We used ROC (receiver operating curve) to assess the diagnostic value of KRT19P3 in BC and normal breast. Chi-square test and Fisher's exact test were used to analyze the relationship between KRT19P3 and clinical parameters as well as PD-L1. The correlation analysis was performed using Spearman's correlation test. Differences between the two groups were analyzed using a T-test. $P$ value $<0.05$ was considered statistically significant.

\section{RESULTS}

\section{LncRNA KRT19P3 Inhibited BC Cell Proliferation, Migration, and Invasion In Vitro}

Regarding the KRT19P3 played an important part in gastric cancer cell migration and invasion capability, we investigated the role of KRT19P3 on BC cells. To reveal the role of KRT19P3 in BC cell proliferation, we overexpressed KRT19P3 with pcDNA3.1(+) plasmid vector or downregulated KRT19P3 using siRNA in MDA-MB-231 cells. The overexpression and knockdown efficiency was confirmed by qRT-PCR, with $24.6 \pm 2.3$-fold increase and $0.5 \pm 0.1$-fold decreases respectively, in MDA-MB231 cells after $24 \mathrm{~h}$ transfection (Figures 1A, B). EdU assays were carried out to examine cell proliferation ability in different groups. The results showed that overexpression of KRT19P3 significantly inhibited MDA-MB-231 cells proliferation by $10.85 \%$ (Figure 1C), whereas, KRT19P3 knockdown enhanced cell proliferation by $12.79 \%$ compared with a control group (Figure 1D). In addition, the transwell assay revealed that upregulation of KRT19P3 substantially increased the migration and invasion rate of MBAMD-231 cells by $45.13 \%$ and $45.45 \%$ respectively (Figure 1E). In contrast, KRT19P3 knockdown remarkably decreased the migration and invasion rate of MBA-MD-231 cells by $38.85 \%$ and $23.69 \%$ respectively (Figure 1F). In conclusion, the results showed that
KRT19P3 functions as a tumor suppressor gene in vitro. These results indicated that KRT19P3 suppressed tumorigenesis via reducing proliferation capacity, migration, and invasion abilities of BC cells in vitro.

\section{LncRNA KRT19P3 Inhibited BC Growth In Vivo}

Vitro experiments showed that KRT19P3 functions as a tumor suppressor gene. We further explored the effect of KRT19P3 on $\mathrm{BC}$ cells in vivo. The results showed that the tumor volumes of LV-KRT19P3 transfected cells were significantly smaller than that of LV-NC transfected xenograft tumors (Figure 2A). Immunohistochemistry for Ki-67 detection revealed that tumor cells in the LV-KRT19P3 group showed a lower positivity rate than those in the LV-NC group (Figure 2B). In summary, these data demonstrated that KRT19P3 suppressed BC in vivo.

\section{LncRNA KRT19P3 Significantly Decreased in Human BC Tissues}

KRT19P3 inhibits the functions of BC cells through the results in vitro and in vivo. We explored the relationship between KRT19P3 and clinical parameters.

Paired specimens were collected from 98 pairs of female BC patients (Figures 3A, B), and the tumor size ranged from 0.60 $\mathrm{cm}$ to $9.00 \mathrm{~cm}$. The RT-qPCR result showed that the median expression of KRT19P3 was 0.0063 in BC tissues and 0.0550 in non-tumor tissues. KRT19P3 was 8.7302-fold higher in para cancer tissues than in tumor tissues (Figure 3C). The ROC curve was drawn according to the expression of KRT19P3 in BC and para cancer tissues and the AUC under the curves was calculated. AUC was 0.9296, indicating that KRT19P3 could better differentiate BC tissues from non-tumor tissues (Figure 3D).

Moreover, IHC results indicated that $\mathrm{Ki}-67$ expression was reduced in the KRT19P3 up-regulated group compared with the control group (Figure 3E). On the other hand, a comparison of clinical parameters showed an inverse relationship between KRT19P3 and pathological grade (Figure 3F).

\section{LncRNA KRT19P3 Correlated With PD-L1 and $\mathrm{CD}^{+} \mathrm{T}$ Cell}

We conducted IHC experiments to detect the positive rate of $\mathrm{PD}$ $\mathrm{L} 1$ and $\mathrm{CD}^{+} \mathrm{T}$ in tissues from 80 pairs of breast cancer patients. Statistical analysis indicated that the expression of PD-L1 was significantly lower (Figures 4A-C) and $\mathrm{CD}^{+} \mathrm{T}$ was significantly higher (Figures 4D-F) in the KRT19P3 high expression group. Spearman analysis revealed that KRT19P3 is negatively correlated with PD-L1 $(r=-0.227, P=0.047)$ and positively correlated with $\mathrm{CD}^{+} \mathrm{T}(r=0.223, P=0.046$, Table 1). The results suggested that KRT19P3 may affect the function of $\mathrm{CD}^{+} \mathrm{T}$ through the PD-1/PD-L1 axis.

\section{DISCUSSION}

Oncogenesis is a gradual process, involving multistage reactions and the accumulation of multiple molecular mutations. 
A

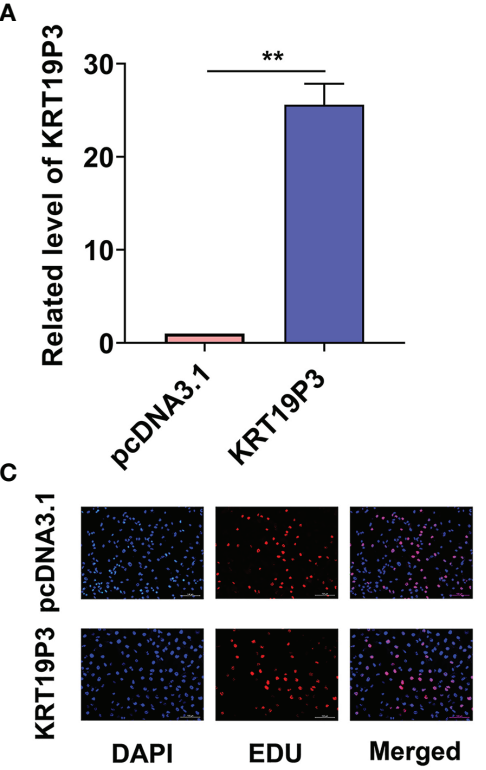

D

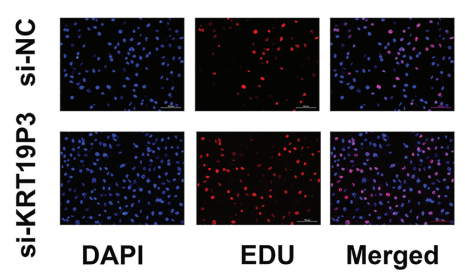

B
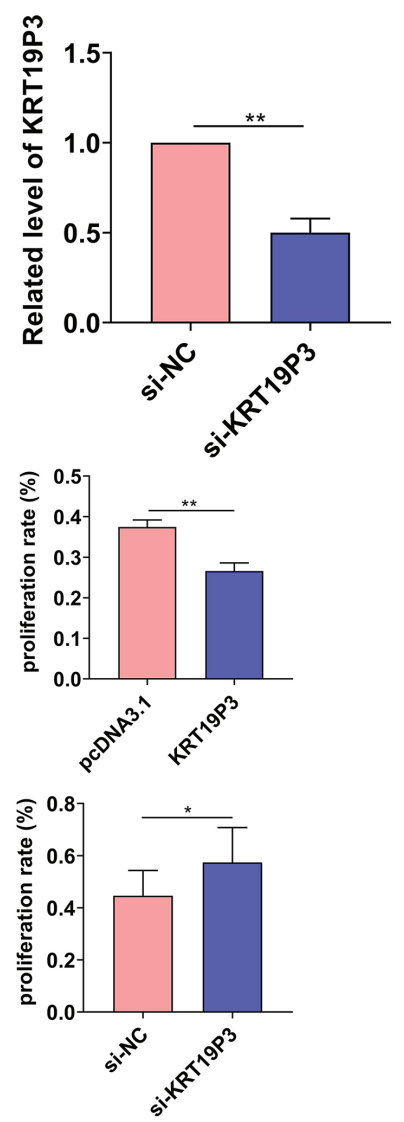

$\mathrm{E}$

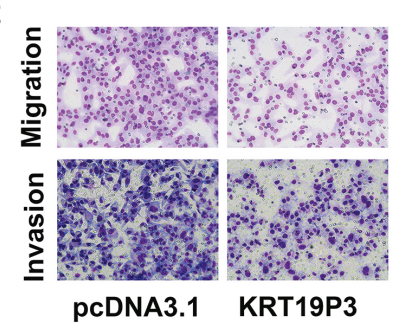

$\mathbf{F}$
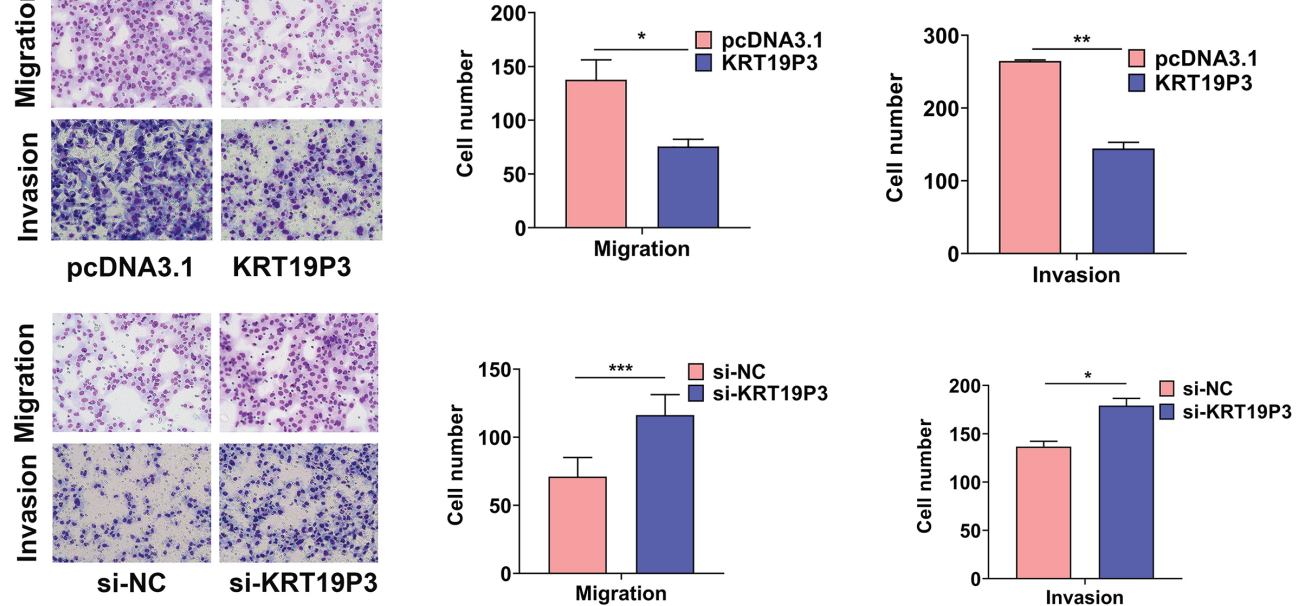

FIGURE 1 | KRT19P3 inhibited BC cell proliferation, migration and invasion. (A) RT-qPCR results showed that pcDNA3.1-KRT19P3 transfection significantly increased the KRT19P3 level in MDA-MB-231 cells. (B) RT-qPCR results showed that siRNA transfection significantly decreased the KRT19P3 level in MDA-MB-231 cells. (C) EDU assay to detect the effect of overexpression of KRT19P3 on cell proliferation. (D) EDU assay to detect the effect of interfering KRT19P3 on cell proliferation. (E) Transwell assay to detect the effect of overexpression of KRT19P3 on cell migration and invasion. (F) Transwell assay to detect the effect of interfering with KRT19P3 on cell migration and invasion. ${ }^{\star} P<0.05,{ }^{\star \star} P<0.01,{ }^{\star \star *} P<0.001$.

LncRNAs have been identified as critical players in oncogenesis and immune response. However, the process of lncRNA involvement in tumor immunity remains incompletely elaborated. KRT19P3, located on chromosome 4, is a transcriptional length of 828 bp of IncRNA, suggesting that it can participate in a variety of biological processes (20).

It was confirmed that KRT19P3 was highly expressed in bladder cancer tissues compared to normal bladder tissues (21). 
A
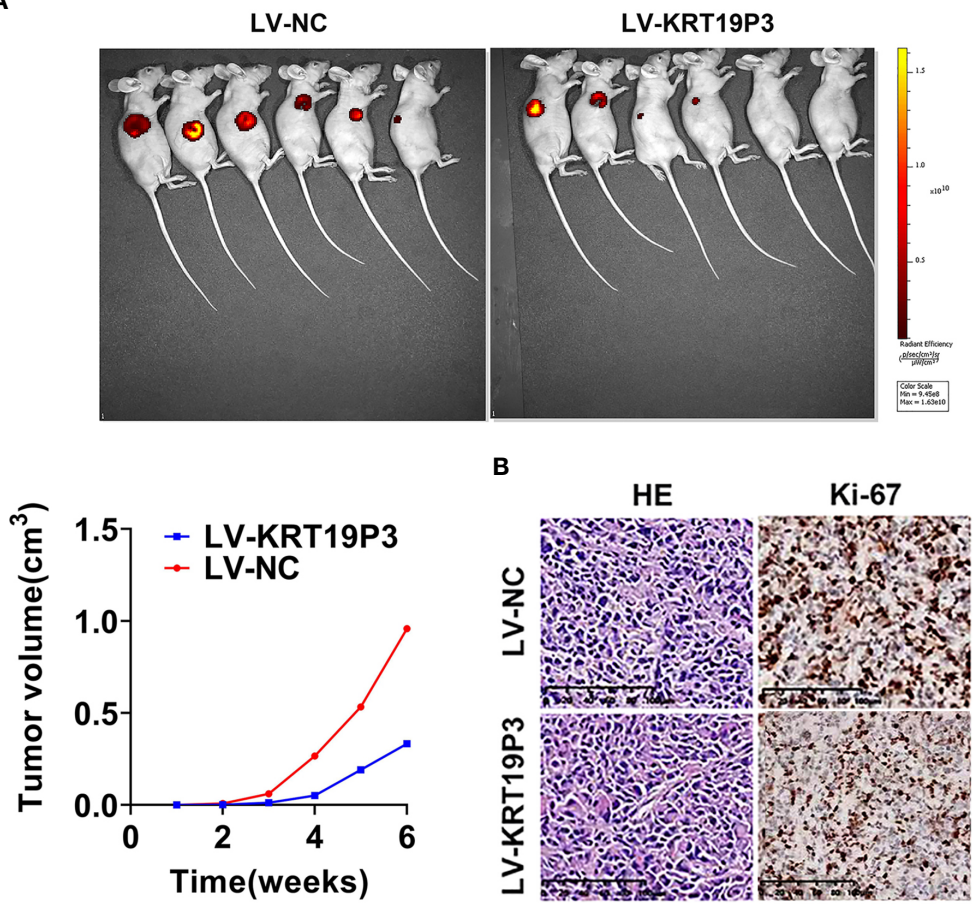

FIGURE 2 | LncRNA KRT19P3 inhibited BC growth in vivo. (A) A stable MDA-MB-231 cell line overexpressing KRT19P3(LV-KRT19P3) was constructed and injected under the subcutaneous of nude mice to establish a xenograft tumor model with 6 mice in each group. Moreover, primary tumor growth was measured every week after injection. The tumor volumes in the LV-KRT19P3 group were significantly smaller than those in the LV-NC group. (B) Immunohistochemistry for Ki-67 detection revealed that cancer cells in the LV-NC group showed a higher positivity rate than those in the LV-KRT19P3 group (magnification $\times 200$ ).

KRT19P3 was less expressed in gastric cancer tissues compared to normal gastric tissues (19). However, the role played by KRT19P3 in the BC is unclear. It is well known that proliferation, migration, and invasion are the obvious biological features of malignant tumors (22). In the present study, we provide evidence for a functional role of lncRNA KRT19P3 in cell proliferation, migration, and invasion of BC. We found that overexpression of KRT19P3 inhibited the proliferation, migration, and invasion of $\mathrm{BC}$ cells in vitro and the growth of xenografts in nude mice. Taken together, KRT19P3 may function as an

Subsequently, we confirmed the differential expression of KRT19P3 in breast cancer tissues and para cancer tissues by clinical samples. The expression of KRT19P3 was higher in nontumor tissues than in BC tissues. The expression of KRT19P3 in $\mathrm{BC}$ tissues and para cancer tissues was plotted by the ROC curve. The result showed that AUC under the curve was 0.9626, indicating that KRT19P3 could be used as a better index to distinguish $\mathrm{BC}$ from normal breast tissues. Correlation analysis with clinical parameters showed that the expression of KRT19P3 in the high Ki-67 index group was lower than that in the low Ki67 index group. It is well known that $\mathrm{Ki}-67$ is an important clinical proliferative marker for many types of cancer, and a high Ki-67 index predicts a poor prognosis for patients (23). Qiu et al. found that the expression of oncogene LINC00668 was positively correlated with the Ki-67 proliferation index in BC tissues, and showed by cell function experiments that downregulation of LINC00668 expression decreased the proliferation of BC cells (24). Similarly, the expression of KRT19P3 in BC tissues was inversely correlated with pathological grade, which is consistent with the finding of a significant positive correlation between the expression of the oncogene lncRNA GClncl and histological grade in bladder cancer tissues found by Zhuang et al. (25). Comprehensive analysis showed that KRT19P3 could inhibit the progression of $\mathrm{BC}$, which further verified the results of in vitro cell experiments and in vivo animal experiments. Meanwhile, the expression of KRT19P3 in BC tissues correlated with the PD-L1 and $\mathrm{CD}^{+} \mathrm{T}$ cells. The results showed that in the group with high KRT19P3 expression, the PD-L1 positivity rate decreased while the $\mathrm{CD}^{+} \mathrm{T}$ cell positivity rate increased. the expression of KRT19P3 was negatively correlated with PD-L1 and positively correlated with CD8+ T cells.

The tumor immune microenvironment is the internal environment for tumors development and metastasis (13, 16, $26,27) . \mathrm{CD}^{+} \mathrm{T}$ cells play an important role in the elimination of tumor cells and their continued proliferation and growth after the elimination of tumor immune escape (28) and are also the main effector cells in the elimination of tumor cells in the internal environment (29). However, PD-L1 is a protein molecule that inhibits the action of effector $\mathrm{T}$ cells and is one of the ligands of PD-1, which is expressed by a variety of tumor cells as well as immune cells. High expression of PD-L1 in BC is 
A
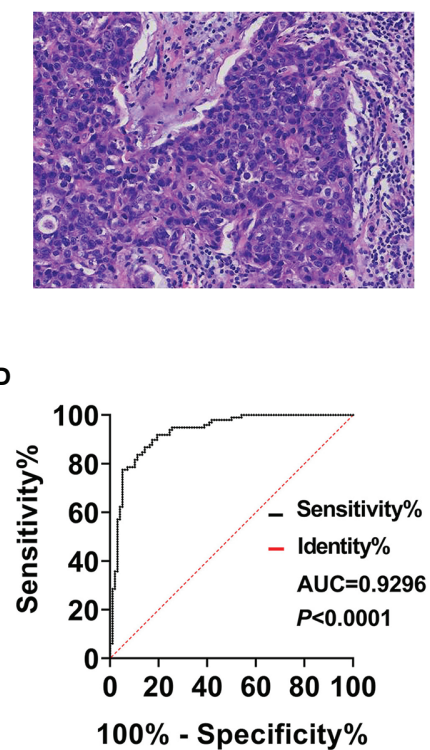

B

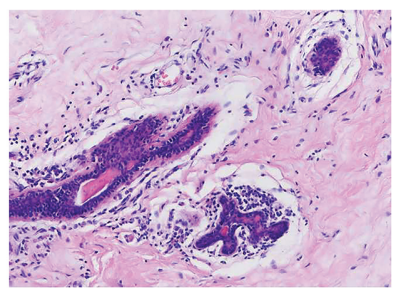

C
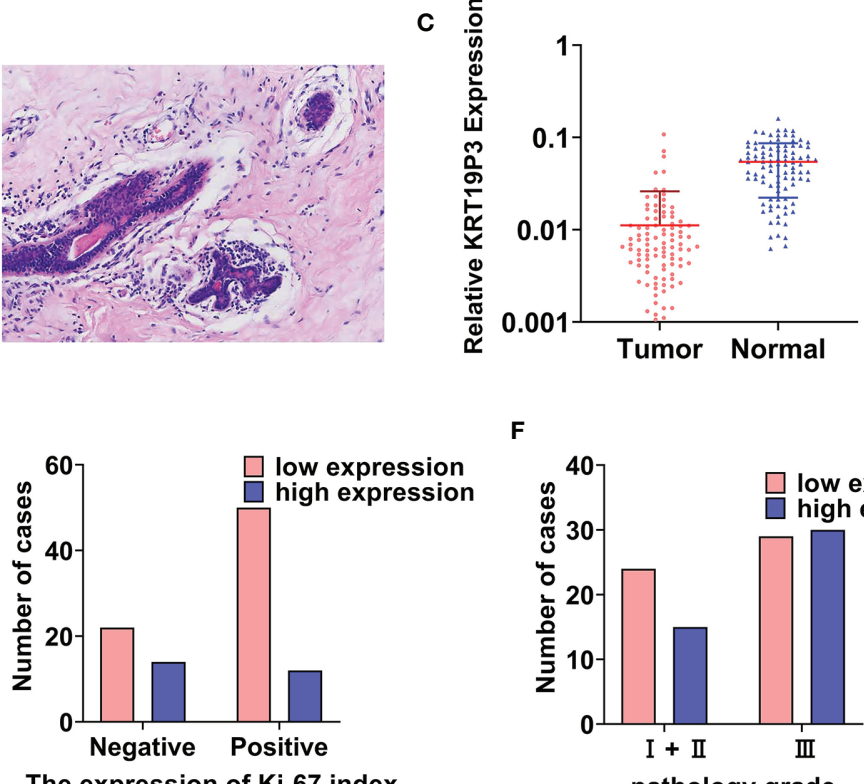

The expression of Ki-67 index

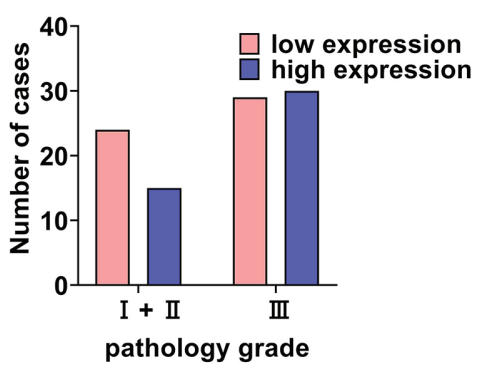

FIGURE 3 | LncRNA KRT19P3 significantly decreased in human BC tissues. (A) HE pictures of breast cancer tissue (H\&E $\times 200)$. (B) HE pictures of normal breast tissue (H\&E $\times 200)$. (C) The expression of KRT19P3 in human breast cancer tissues (Tumor, $n=98$ ) and corresponding para cancer tissues (Normal, $n=98)$ was detected by RT-qPCR. (D) The ROC curve was drawn according to the expression of KRT19P3 in breast cancer tissues and para cancer tissues (AUC=0.9296, $P<0.0001)$. (E) Relationship between KRT19P3 and Ki-67 index (Ki-67 positive rate $\geq 30 \%, r=-0.213, P<0.05)$. (F) Relationship between KRT19P3 and pathology grade $(r=-0.227, P<0.05)$.

A

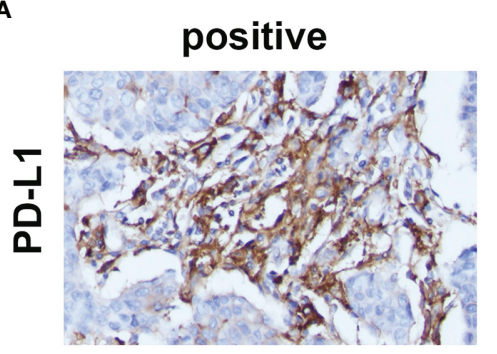

D

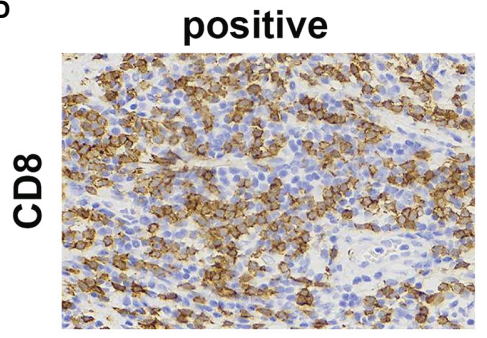

B

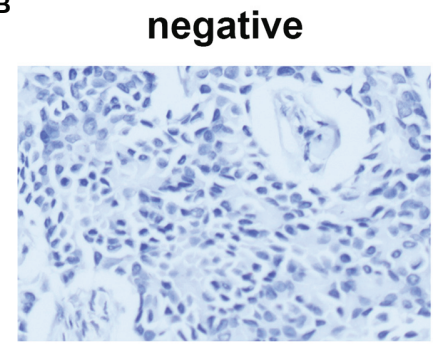

E

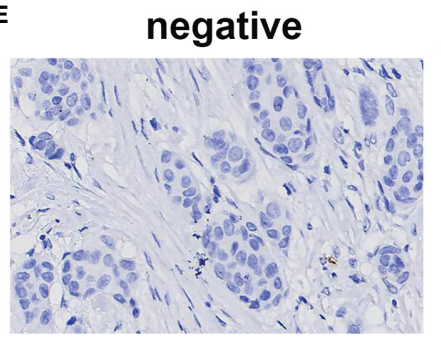

c

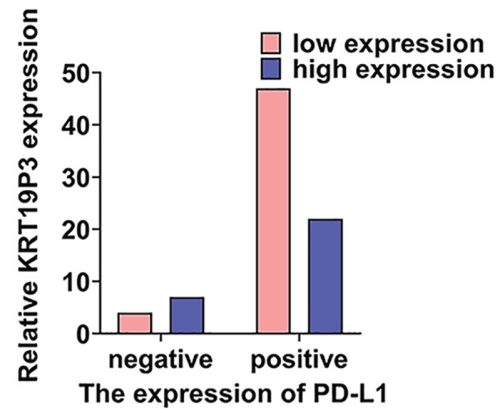

F

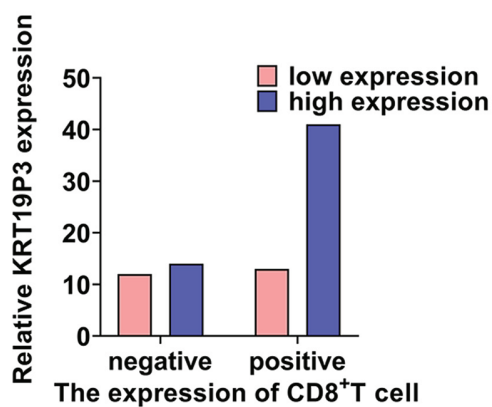

FIGURE 4 | LncRNA KRT19P3 correlated with PD-L1 and CD8 ${ }^{+}$T cell. (A, B) PD-L1 expression in breast cancer tissue (A positive staining, 200x; B negative staining, 200x). (C) KRT19P3 expression was lower in the PD-L1-positive group than in the PD-L1-negative group in breast cancer tissues (n=80). (D, E) CD8 expression in breast cancer tissues (D positive staining, 200x, E negative staining, 200x). (F) KRT19P3 expression was higher in the CD8 ${ }^{+} \mathrm{T}$ high group than in the $\mathrm{CD}^{+} \mathrm{T}$ cell low expression group in breast cancer tissues $(\mathrm{n}=80)$. 
TABLE 1 | The relationship between KRT19P3 and PD-L1, CD8 in BC tissues.

\begin{tabular}{|c|c|c|c|c|c|}
\hline & \multirow[t]{2}{*}{ Number } & \multicolumn{2}{|c|}{ KRT19P3 } & \multirow[t]{2}{*}{$\chi^{2}$} & \multirow[t]{2}{*}{ P-value } \\
\hline & & Low expression & High expression & & \\
\hline PD-L1 & & & & 4. 139 & $0.047^{\star}$ \\
\hline Negative & 11 & 4 & 7 & & \\
\hline Positive & 69 & 47 & 22 & & \\
\hline CD8 & & & & 3. 982 & $0.046^{\star}$ \\
\hline Negative & 26 & 12 & 14 & & \\
\hline Positive & 54 & 13 & 41 & & \\
\hline
\end{tabular}

associated with prognostic markers of malignancy $(30,31)$. When PD-1 binds to its receptor, PD-L1, it transmits negative regulatory signals to $\mathrm{T}$ cells and induces them to become dormant. Based on the correlation analysis between KRT19P3 and PD-L1, CD8 ${ }^{+}$T, it was hypothesized that KRT19P3 may inhibit $\mathrm{BC}$ progression by reducing $\mathrm{PD}-\mathrm{L} 1$ expression in tumor cells and activating the tumor-killing potential of $\mathrm{CD}^{+} \mathrm{T}$ cells, which is also consistent with the findings of Sun et al. (32), who blocked the PD-1/PD-L1 pathway and thus rescued the depleted $\mathrm{CD}^{+} \mathrm{T}$ cells.

In summary, our study confirmed that KRT19P3 was less expressed in $\mathrm{BC}$ than in normal breast tissues and inhibited the function of BC cells. Combined with clinical parameters, KRT19P3 played the role of the tumor suppressor gene in BC. The expression of KRT19P3 was negatively correlated with PDL1 but positively correlated with $\mathrm{CD}^{+} \mathrm{T}$ cells. Therefore, KRT19P3 may inhibit BC progression through the immune pathway. We will actively explore the specific mechanism in

\section{REFERENCES}

1. He B, Zhang Y, Zhou Z, Wang B, Liang Y, Lang J, et al. A Neural Network Framework for Predicting the Tissue-Of-Origin of 15 Common Cancer Types Based on RNA-Seq Data. Front Bioeng Biotechnol (2020) 8:737. doi: 10.3389/ fbioe.2020.00737

2. Zhuang J, Dai S, Zhang L, Gao P, Han Y, Tian G, et al. Identifying Breast CancerInduced Gene Perturbations and Its Application in Guiding Drug Repurposing. Curr Bioinf (2020) 15:1075-89. doi: 10.2174/1574893615666200203104214

3. Sung H, Ferlay J, Siegel RL, Laversanne M, Soerjomataram I, Jemal A, et al. Global Cancer Statistics 2020: GLOBOCAN Estimates of Incidence and Mortality Worldwide for 36 Cancers in 185 Countries. CA: A Cancer J Clin (2021) 71:209-49. doi: 10.3322/caac.21660

4. Mathias C, Zambalde EP, Rask P, Gradia DF, De Oliveira JC. Long NonCoding RNAs Differential Expression in Breast Cancer Subtypes: What Do We Know? Clin Genet (2019) 95:558-68. doi: 10.1111/cge.13502

5. Amer A, Nagah A, Tian T, Zhang X. Mutation Mechanisms of Breast Cancer Among the Female Population in China. Curr Bioinf (2020) 15:253-9. doi: $10.2174 / 1574893615666191220141548$

6. Zhang Y, Xiang J, Tang L, Li J, Lu Q, Tian G, et al. Identifying Breast CancerRelated Genes Based on a Novel Computational Framework Involving KEGG Pathways and PPI Network Modularity. Front Genet (2021) 12:596794. doi: 10.3389/fgene.2021.596794

7. Kung JT, Colognori D, Lee JT. Long Noncoding RNAs: Past, Present, and Future. Genetics (2013) 193:651-69. doi: 10.1534/genetics.112.146704

8. Chen X, You ZH, Yan GY, Gon DW. IRWRLDA: Improved Random Walk With Restart for lncRNA-Disease Association Prediction. Oncotarget (2016) 7:540334-54041. doi: 10.18632/oncotarget.11141 the follow-up study. This finding may provide a new direction for the diagnosis and treatment of BC.

\section{DATA AVAILABILITY STATEMENT}

The original contributions presented in the study can be directed to the corresponding author.

\section{ETHICS STATEMENT}

The animal study was reviewed and approved by Ethics Committee of Weifang People's Hospital.

\section{AUTHOR CONTRIBUTIONS}

YZ: guarantor of integrity of the entire study, study concepts, and manuscript editing. YF, XD, and ML: study design, manuscript review, clinical sample collection, experimental procedures, and statistical analysis. PL: English Language Embellishment. JZ and HL: resources, laboratory samples, and instrumentation supplied. All authors read and approved the final manuscript.

\section{ACKNOWLEDGMENTS}

All authors were informed about publishing the results and they all agreed.

9. Ji J, Tang J, Xia K-J, Jiang R. LncRNA in Tumorigenesis Microenvironment. Curr Bioinf (2019) 14:640-1. doi: 10.2174/157489361407190917161654

10. Feng F, Chen A, Huang J, Xia Q, Chen Y, Jin X. Long Noncoding RNA SNHG16 Contributes to the Development of Bladder Cancer via Regulating miR-98/STAT3/Wnt/beta-Catenin Pathway Axis. J Cell Biochem (2018) 119:9408-18. doi: 10.1002/jcb.27257

11. Xiao X, Zhu W, Liao B, Xu J, Gu C, Ji B, et al. BPLLDA: Predicting lncRNADisease Associations Based on Simple Paths With Limited Lengths in a Heterogeneous Network. Front Genet (2018) 9:411. doi: 10.3389/ fgene.2018.00411

12. Huang PS, Lin YH, Chi HC, Tseng YH, Chen CY, Lin TK, et al. Dysregulated FAM215A Stimulates LAMP2 Expression to Confer Drug-Resistant and Malignant in Human Liver Cancer. Cells (2020) 9:961. doi: 10.3390/ cells9040961

13. Ran W, Chen X, Wang B, Yang P, Li Y, Xiao Y, et al. Whole-Exome Sequencing of Tumor-Only Samples Reveals the Association Between Somatic Alterations and Clinical Features in Pancreatic Cancer. Curr Bioinf (2020) 15:1160-7. doi: 10.2174/1574893615999200626190346

14. Dai W, Zeng W, Lee D. lncRNA MCM3AP-AS1 Inhibits the Progression of Colorectal Cancer via the miR-19a-3p/FOXF2 Axis. J Gene Med (2021) 23: e3306. doi: 10.1002/jgm.3306

15. Mowel WK, Kotzin JJ, Mccright SJ, D Neal V, Henao-Mejia J. Control of Immune Cell Homeostasis and Function by lncRNAs. Trends Immunol (2018) 39:55-69. doi: 10.1016/j.it.2017.08.009

16. Chen QH, Li B, Liu DG, Zhang B, Yang X, Tu YL. LncRNA KCNQ1OT1 Sponges miR-15a to Promote Immune Evasion and Malignant Progression of Prostate Cancer via Up-Regulating PD-L1. Cancer Cell Int (2020) 20:394. doi: 10.1186/s12935-020-01481-8 
17. Ai Y, Wu S, Gao H, Wei H, Tang Z, Li X, et al. Repression of CRNDE Enhances the Anti-Tumour Activity of CD8 + T Cells Against Oral Squamous Cell Carcinoma Through Regulating miR-545-5p and TIM-3. J Cell Mol Med (2021) 25:10857-10868. doi: 10.1111/jcmm.16909

18. Rech AJ, Vonderheide RH. Dynamic Interplay of Oncogenes and T Cells Induces PD-L1 in the Tumor Microenvironment. Cancer Discovery (2013) 3:1330-2. doi: 10.1158/2159-8290.CD-13-0775

19. Zheng J, Zhang H, Ma R, Liu H, Gao P. Long non-Coding RNA KRT19P3 Suppresses Proliferation and Metastasis Through COPS7A-Mediated NFkappaB Pathway in Gastric Cancer. Oncogene (2019) 38:7073-88. doi: 10.1038/s41388-019-0934-Z

20. Wang S, Liu X. The UCSCXenaTools R Package: A Toolkit for Accessing Genomics Data From UCSC Xena Platform, From Cancer Multi-Omics to Single-Cell RNASeq. J Open Source Software (2019) 4:1627. doi: 10.21105/joss.01627

21. Zhu YP, Bian XJ, Ye DW, Yao XD, Zhang SL, Dai B, et al. Long Noncoding RNA Expression Signatures of Bladder Cancer Revealed by Microarray. Oncol Lett (2014) 7:1197-202. doi: 10.3892/ol.2014.1843

22. Wan Q, Tang M, Sun S-L, Hu J, Sun Z-J, Fang Y-T, et al. SNHG3 Promotes Migration, Invasion, and Epithelial-Mesenchymal Transition of Breast Cancer Cells Through the miR-186-5p/ZEB1 Axis. Am J Transl Res (2021) 13:585-600.

23. Li L, Han D, Wang X, Wang Q, Tian J, Yao J, et al. Prognostic Values of Ki-67 in Neoadjuvant Setting for Breast Cancer: A Systematic Review and MetaAnalysis. Future Oncol (2017) 13:1021-34. doi: 10.2217/fon-2016-0428

24. Li J, Zhai Q, Huang HL, Chen Z, Zhang QF. LncRNA DCST1-AS1 Accelerates the Proliferation, Metastasis and Autophagy of Hepatocellular Carcinoma Cell by AKT/mTOR Signaling Pathways. Eur Rev Med Pharmacol Sci (2019) 23:6091-104. doi: 10.26355/eurrev_201907_18423

25. Zhuang C, Ma Q, Zhuang C, Ye J, Zhang F, Gui Y. LncRNA GClncl Promotes Proliferation and Invasion of Bladder Cancer Through Activation of MYC. FASEB J (2019) 33:11045-59. doi: 10.1096/fj.201900078RR

26. Tang W, Wan S, Yang Z, Teschendorff AE, Zou Q. Tumor Origin Detection With Tissue-Specific miRNA and DNA Methylation Markers. Bioinformatics (2018) 34:398-406. doi: 10.1093/bioinformatics/btx622

27. Liu H, Qiu C, Wang B, Bing P, Tian G, Zhang X, et al. Evaluating DNA Methylation, Gene Expression, Somatic Mutation, and Their Combinations in
Inferring Tumor Tissue-Of-Origin. Front Cell Dev Biol (2021) 9:619330. doi: $10.3389 /$ fcell.2021.619330

28. Farhood B, Najafi M, Mortezaee K. CD8(+) Cytotoxic T Lymphocytes in Cancer Immunotherapy: A Review. J Cell Physiol (2019) 234:8509-21. doi: $10.1002 /$ jcp. 27782

29. Mahmoud SM, Paish EC, Powe DG, Macmillan RD, Grainge MJ, Lee AH, et al. Tumor-Infiltrating CD8+ Lymphocytes Predict Clinical Outcome in Breast Cancer. J Clin Oncol (2011) 29:1949-55. doi: 10.1200/ JCO.2010.30.5037

30. Sabatier R, Finetti P, Mamessier E, Adelaide J, Chaffanet M, Ali HR, et al. Prognostic and Predictive Value of PDL1 Expression in Breast Cancer. Oncotarget (2014) 6:5449-64. doi: 10.18632/oncotarget.3216

31. Hou Y, Nitta H, Parwani AV, Li Z. PD-L1 and CD8 are Associated With Deficient Mismatch Repair Status in Triple-Negative and HER2-Positive Breast Cancers. Hum Pathol (2019) 86:108-14. doi: 10.1016/ j.humpath.2018.12.007

32. Sun C, Mezzadra R, Schumacher TN. Regulation and Function of the PD-L1 Checkpoint. Immunity (2018) 48:434-52. doi: 10.1016/j.immuni.2018.03.014

Conflict of Interest: The authors declare that the research was conducted in the absence of any commercial or financial relationships that could be construed as a potential conflict of interest.

Publisher's Note: All claims expressed in this article are solely those of the authors and do not necessarily represent those of their affiliated organizations, or those of the publisher, the editors and the reviewers. Any product that may be evaluated in this article, or claim that may be made by its manufacturer, is not guaranteed or endorsed by the publisher.

Copyright (C) 2022 Fan, Dong, Li, Liu, Zheng, Li and Zhang. This is an open-access article distributed under the terms of the Creative Commons Attribution License (CC BY). The use, distribution or reproduction in other forums is permitted, provided the original author(s) and the copyright owner(s) are credited and that the original publication in this journal is cited, in accordance with accepted academic practice. No use, distribution or reproduction is permitted which does not comply with these terms. 